SFB

A cautionary note on computing conditional from unconditional correlations

823

Jonas Kaiser, Walter Krämer

Nr. 8/2009

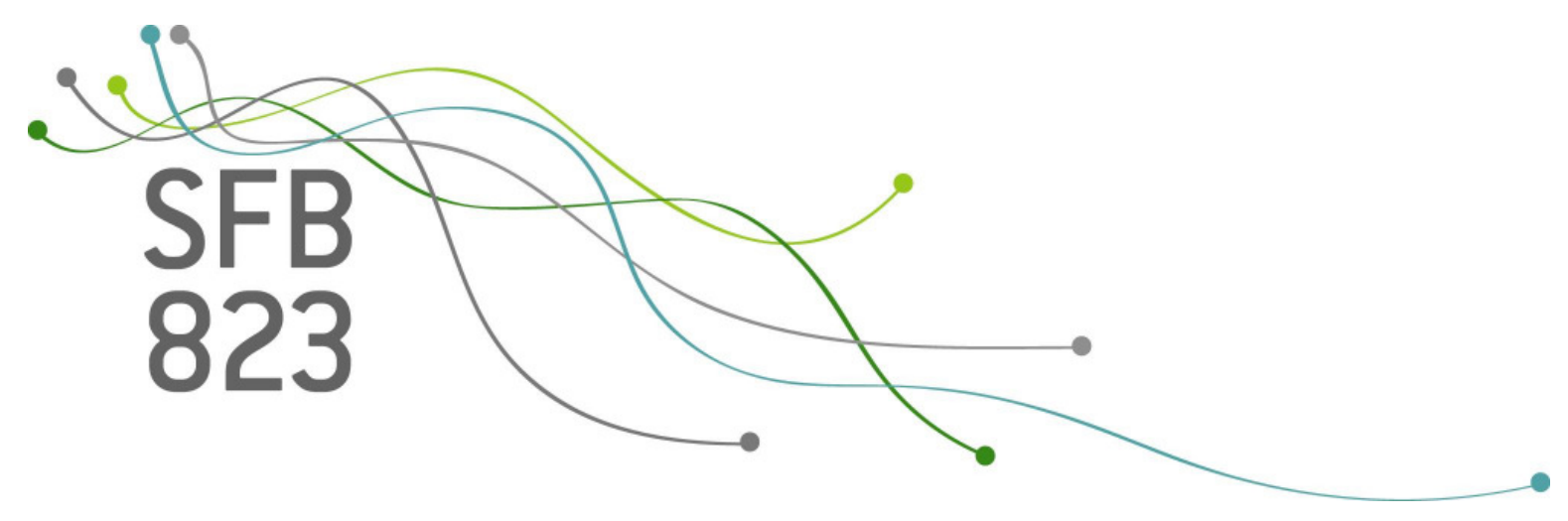





\title{
A cautionary note on computing conditional from unconditional correlations ${ }^{1}$
}

\author{
by \\ Jonas Kaiser \\ Fakultät Statistik, Technische Universität Dortmund, Germany \\ Phone xx231/755-3125, Fax: xx231/755-5284 \\ e-mail: kaiser@statistik.tu-dortmund.de \\ and \\ Walter Krämer \\ Fakultät Statistik, Technische Universität Dortmund, Germany \\ Phone xx231/755-3125, Fax: xx231/755-5284 \\ e-mail: walterk@statistik.tu-dortmund.de \\ version January 2009
}

\begin{abstract}
Summary
We show that some care should be exercised when inferring true unconditional correlations from observed conditional correlations, which is a frequent problem in empirical finance and elsewhere. We give a general formula for the relationship between the two and demonstrate its importance in the context of the bivariate $t$ distribution.
\end{abstract}

Keywords: conditional correlation, $t$-distribution, stock returns

JEL-numbers: C13, C21, C34

\footnotetext{
${ }^{1}$ Research supported by Deutsche Forschungsgemeinschaft (DFG)
} 


\section{The problem}

Figure 1 shows 2524 daily stock returns (from 1998 to 2008) of DaimlerChrysler and Deutsche Bank. Panel (a) shows the unconditional empirical distribution, with a correlation coefficient $\hat{\rho}=0.61$, and panel (b) shows the conditional distribution, given that Daimler-Chrysler returns are larger than 2 percent in absolute value. Here, the empirical correlation coefficient is $\hat{\rho}=0.71$.

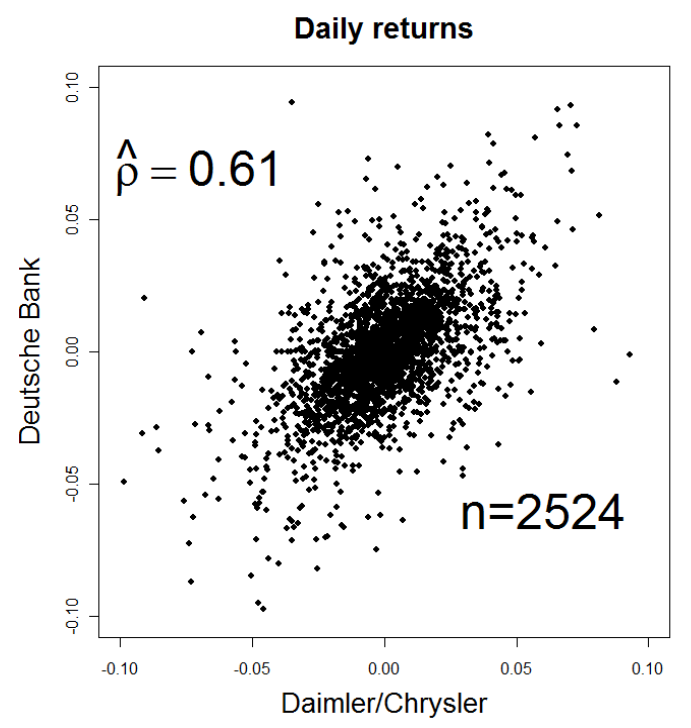

(a)

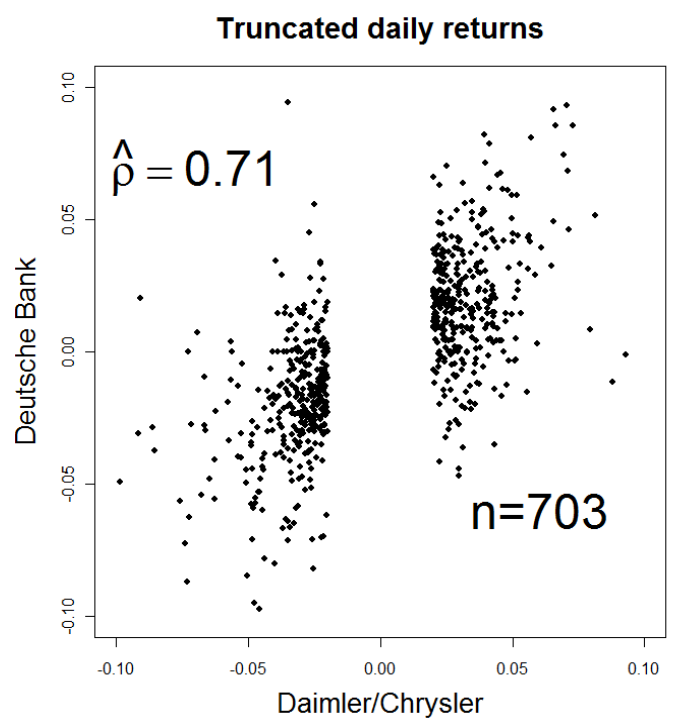

(b)

Figure 1: Daily returns of Daimler-Chrysler and Deutsche Bank

This change in empirical correlation, given certain conditions like the one above, has been the subject of quite some debate in empirical finance recently (Longin and Solnik 1995, Boyer et al. 1997, Forbes and Rigobon 2002, Campbell et al. 2008, among many others). As a result, it has become clear that considerable care should be taken when inferring true population correlations from observed conditional ones. Another example, different from the one we consider here, occurs when comparing correlations of returns and other quanti- 
ties in volatile and tranquil periods, see Solnik et al. (1996) or Bautista (2006), who presents an application to exchange rate-interest differentials. For bivariate normal random variables $X$ and $Y$ with unconditional correlation $\rho$, it is well known (Johnson and Kotz 1972, Boyer et al. 1999) that the conditional correlation of $X$ and $Y$, given $X \in A, 0<P(A)<1$, is

$$
\rho_{X Y \mid X \in A}=\frac{\rho}{\sqrt{\rho^{2}+\left(1-\rho^{2}\right) \frac{\sigma_{X}^{2}}{\sigma_{X \mid X \in A}^{2}}}} .
$$

This implies that $\left|\rho_{A}\right|>|\rho|$ if $\sigma_{X \mid X \in A}^{2}>\sigma_{X}^{2}$, which for instance occurs whenever one conditions on large absolute values of $X$.

As neither uni- nor multivariate normality can safely be assumed for the returns of risky assets, there is some interest in extending this formula to more realistic distributions. This is done here, with an application to the bivariate $t$-distribution, following Campbell et al. (2008). However, other than Campbell et al., we do not construct bivariate $t$-variables from independent marginals.

\section{A general theorem}

For ease of notation, we will write $\rho_{X Y ; A}$ instead of $\rho_{X Y \mid X \in A}, \sigma_{X ; A}^{2}$ instead of $\sigma_{X \mid X \in A}^{2}$ and so on.

Now, let $X$ and $Y$ be any two random variables with finite second moments and correlation $\rho_{X Y}=\sigma_{X Y} / \sigma_{X} \sigma_{Y}$, where, without loss of generality, we assume $\mathrm{E}(X)=\mathrm{E}(Y)=0$ and $\sigma_{Y}=1 . Y$ can then be expressed as

$$
Y=\frac{\rho_{X Y}}{\sigma_{X}} X+\sqrt{1-\rho_{X Y}^{2}} Z,
$$


where $Z$ is a random variable with unit variance computed from $X$ and $Y$ such that $Z$ is uncorrelated with $X$ :

$$
Z=-\frac{\rho}{\sqrt{1-\rho^{2}}} \frac{X}{\sigma_{X}}+\frac{1}{\sqrt{1-\rho^{2}}} Y .
$$

The conditional correlation $\rho_{X Y ; A}$ of $X$ and $Y$, given $X \in A, 0<P(A)<1$, now hinges crucially on the behaviour of this auxiliary variable $Z$, given $X \in A$.

Theorem: If $X$ and $Z$ remain uncorrelated given $X \in A$, i.e. if $\sigma_{X Z ; A}=0$, we have

$$
\rho_{X Y ; A}=\frac{\rho_{X Y}}{\sqrt{\rho_{X Y}^{2}+\frac{\left(1-\rho_{X Y}^{2}\right) \sigma_{X}^{2} \sigma_{Z ; A}^{2}}{\sigma_{X ; A}^{2}}}} .
$$

Proof:

$$
\begin{aligned}
\rho_{X Y ; A} & =\frac{\sigma_{X Y ; A}}{\sigma_{X ; A} \sigma_{Y ; A}} \\
& =\frac{\operatorname{Cov}\left(X, \frac{\rho_{X Y}}{\sigma_{X}} X+\sqrt{1-\rho_{X Y}^{2} Z} ; A\right)}{\sigma_{X ; A} \sqrt{\operatorname{Var}\left(\frac{\rho_{X Y}}{\sigma_{X}} X+\sqrt{1-\rho_{X Y}^{2}} Z ; A\right)}} \\
& =\frac{\frac{\rho_{X Y}}{\sigma_{X}} \sigma_{X ; A}^{2}+0}{\sigma_{X ; A} \sqrt{\frac{\rho_{X Y}^{2}}{\sigma_{X}^{2}} \sigma_{X ; A}^{2}+\left(1-\rho_{X Y}^{2}\right) \sigma_{Z ; A}^{2}}} \\
& =\frac{\frac{\rho_{X Y}}{\sigma_{X}}}{\sqrt{\frac{\rho_{X Y}^{2}}{\sigma_{X}^{2}}+\frac{\left(1-\rho_{X Y}^{2}\right) \sigma_{Z ; A}^{2}}{\sigma_{X ; A}^{2}}}} \\
& =\frac{\rho_{X Y}}{\sqrt{\rho_{X Y}^{2}+\frac{\left(1-\rho_{X Y}^{2}\right) \sigma_{X}^{2} \sigma_{Z ; A}^{2}}{\sigma_{X ; A}^{2}}}}
\end{aligned}
$$


The crucial condition $\sigma_{X Z ; A}=0$ is satisfied for instance whenever the joint density of $X$ and the auxiliary variable $Z$ is symmetric with respect to the x-axis. As $X$ and $Z$ are uncorrelated by construction, this is guaranteed for instance for all spherical distributions, in particular for bivariate uncorrelated $t$-variables as defined in section 3 .

\section{Application to the bivariate $t$-distribution}

There is no unique definition of multivariate $t$-distributions. For an overview see Kotz and Nadarajah (2004). Most often, a bivariate random variable $(X, Y)^{\prime}$ is said to be $t$-distributed with $\nu$ degrees of freedom, mean vector $\mu=\left(\mu_{x}, \mu_{y}\right)^{\prime}$ and correlation matrix

$$
R=\left(\begin{array}{cc}
1 & \rho_{X Y} \\
\rho_{X Y} & 1
\end{array}\right)
$$

(in shorthand : $(X, Y)^{\prime} \sim B V T_{\nu}(\mu, R)$ ) if its joint density is given by

$$
\begin{aligned}
f(x, y)= & \frac{1}{2 \pi \sqrt{1-\rho_{X Y}^{2}}} \\
& {\left[1+\frac{\left(x-\mu_{x}\right)^{2}-2 \rho_{X Y}\left(x-\mu_{x}\right)\left(y-\mu_{y}\right)+\left(y-\mu_{y}\right)^{2}}{1-\rho_{X Y}^{2}}\right]^{(-\nu / 2-1)} . }
\end{aligned}
$$

In the following, we assume $\mu=0$ and $\nu>2$ so that the first two moments exist. The bivariate $t$-distribution is spherical if $\rho_{X Y}=0$ and elliptical otherwise. The marginal densities of a $B V T_{\nu}$-variable are univariate $t_{\nu}$.

From Kotz and Nadarajah (2004, p.15), we know in addition that for $(X, Y)^{\prime} \sim B V T_{\nu}(0, R)$ and some scalar nonsingular $(2 \mathrm{x} 2)$-matrix $C$,

$$
C\left(\begin{array}{l}
\mathrm{X} \\
\mathrm{Y}
\end{array}\right) \sim B V T_{\nu}\left(0, C R C^{\prime}\right)
$$


Taking

$$
C=\left(\begin{array}{cc}
1 & 0 \\
-\frac{\rho}{\sqrt{1-\rho^{2}}} & \frac{1}{\sqrt{1-\rho^{2}}}
\end{array}\right)
$$

we have $C R C^{\prime}=I$, so

$$
C\left(\begin{array}{l}
\mathrm{X} \\
\mathrm{Y}
\end{array}\right):=\left(\begin{array}{l}
\mathrm{X} \\
\mathrm{Z}
\end{array}\right) \sim B V T(0, I)
$$

where

$$
Z=-\frac{\rho}{\sqrt{1-\rho^{2}}} X+\frac{1}{\sqrt{1-\rho^{2}}} Y .
$$

The joint density of $X$ and $Z$ is spherical and therefore symmetric with respect to the $x$-axis, so our theorem applies, and, in view of $\operatorname{Var}(X)=\operatorname{Var}(Y)=1$, our expression (2) simplifies to

$$
\rho_{X Y ; A}=\frac{\rho_{X Y}}{\sqrt{\rho_{X Y}^{2}+\frac{\left(1-\rho_{X Y}^{2}\right) \sigma_{Z ; A}^{2}}{\sigma_{X ; A}^{2}}}} .
$$

This differs from (1) which for instance is used by Campbell et al. (2008). The latter formula gives the true correlation of $X$ and

$$
Y=\rho X+\sqrt{1-\rho^{2}} Z
$$

whenever $X$ and the auxiliary variable $Z$ are independent. However, when $X$ and $Z$ are independent $t$-variables, $(X, Y)^{\prime}$ is not bivariate $t$ ! In fact, $Y$ is not even univariate $t$, as shown in figure 2 .

Figure 3 considers joint distributions and plots 5000 observations each of a $B V T_{5}(0, I)$ (panel (a)) and a bivariate vector $(X, Z)^{\prime}$ with independent $t_{5^{-}}$ variables (panel (b)). It is obvious from panel (b) that the joint distribution of $X$ and $Y$ is not spherical for independent marginals. In fact, it is well known 


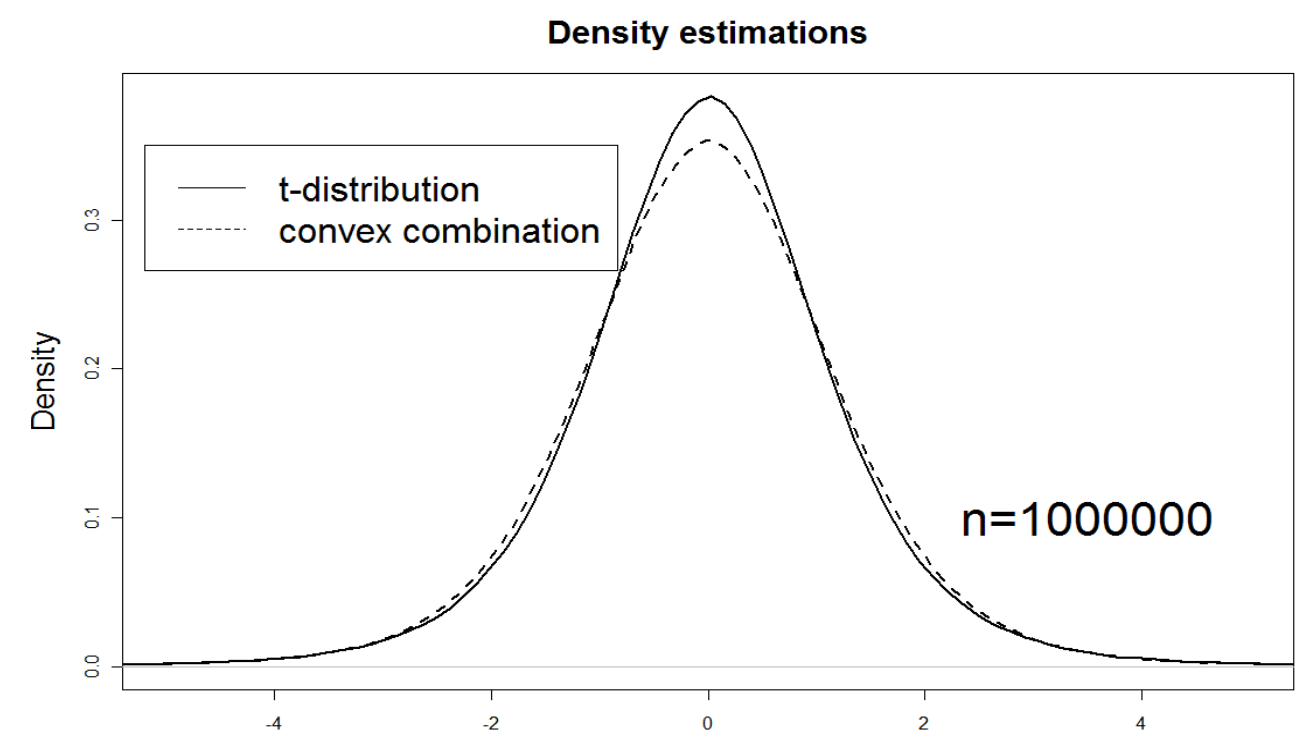

Figure 2: Density estimations of a $t_{5}$-Variable and a convex combination of two independent $t_{5}$-variables

that the multivariate normal distribution is the only one where independence and a spherical density go together (see e.g. Bilodeau and Brenner 1999). Figure 4 plots the respective bivariate distributions of $X$ and $Y$ obtained from multiplying $(X, Z)^{\prime}$ with the matrix $C$ from (3), using $\rho=0.6$. Again, it is obvious from panel $\mathrm{b}$ that the joint distribution of $X$ and $Y$ is not bivariate $t$ if $Y$ is computed from independent $X$ and $Z$. Or, to put it differently: While Campbell et al. pretend to evaluate conditional correlations for random vectors with joint distribution as in panel (a), what they really do is evaluate conditional correlation for random vectors with joint distribution as in panel (b). 


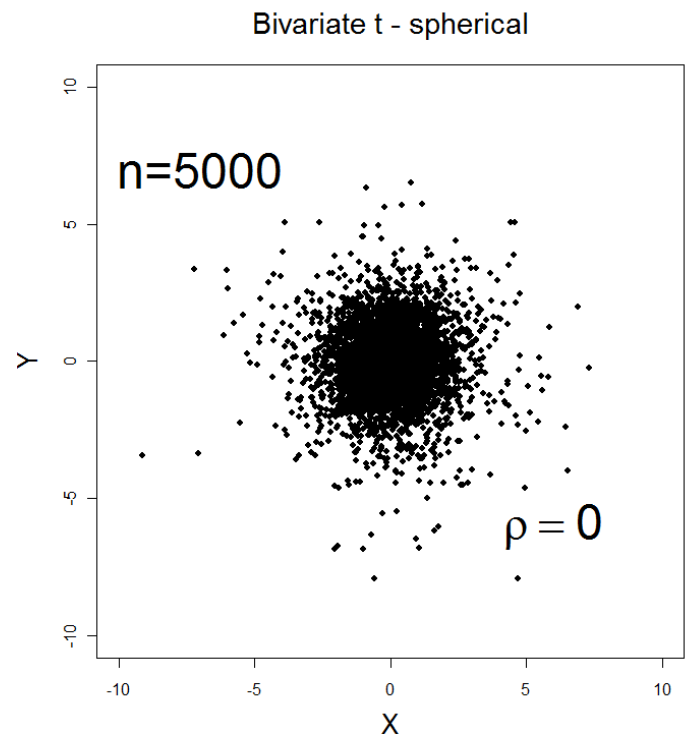

(a)

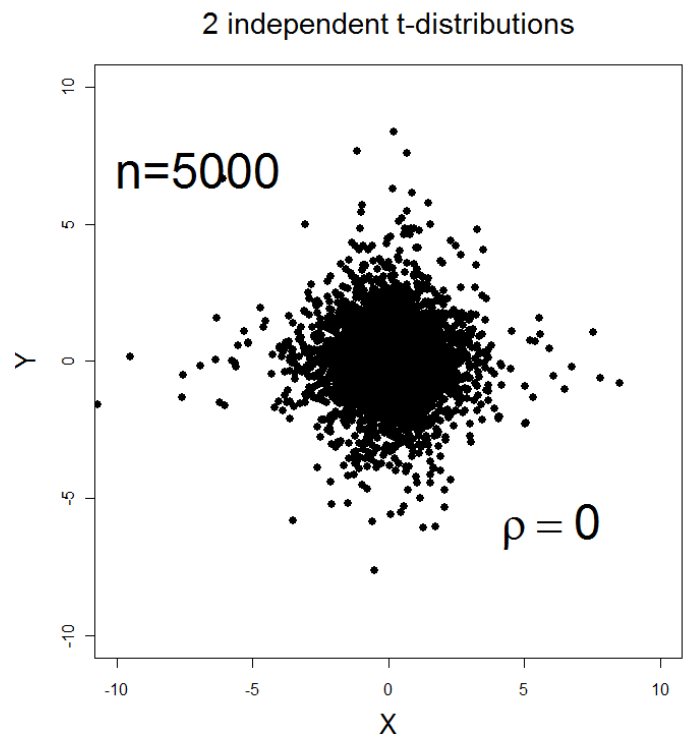

(b)

Figure 3: Joint distribution of uncorrelated(a) and independent (b) $t_{5}$-Variables

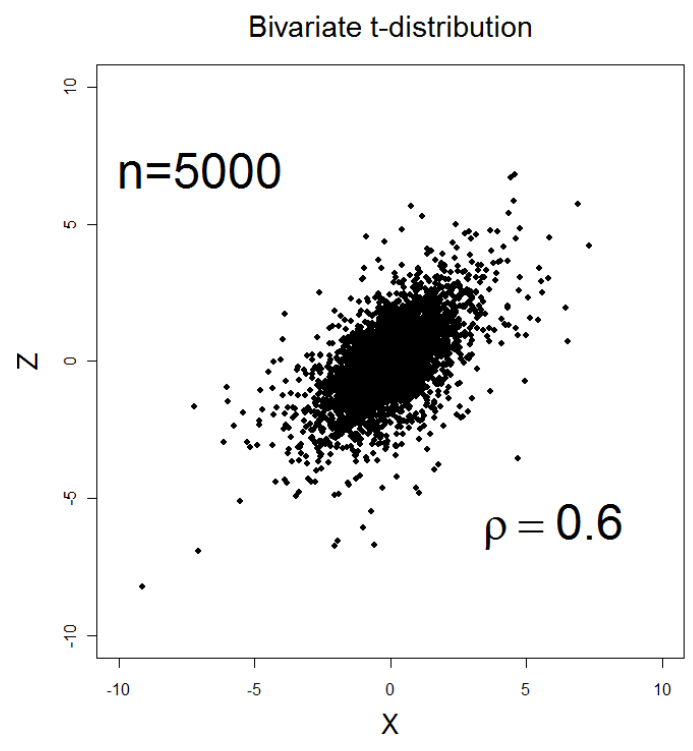

(a)

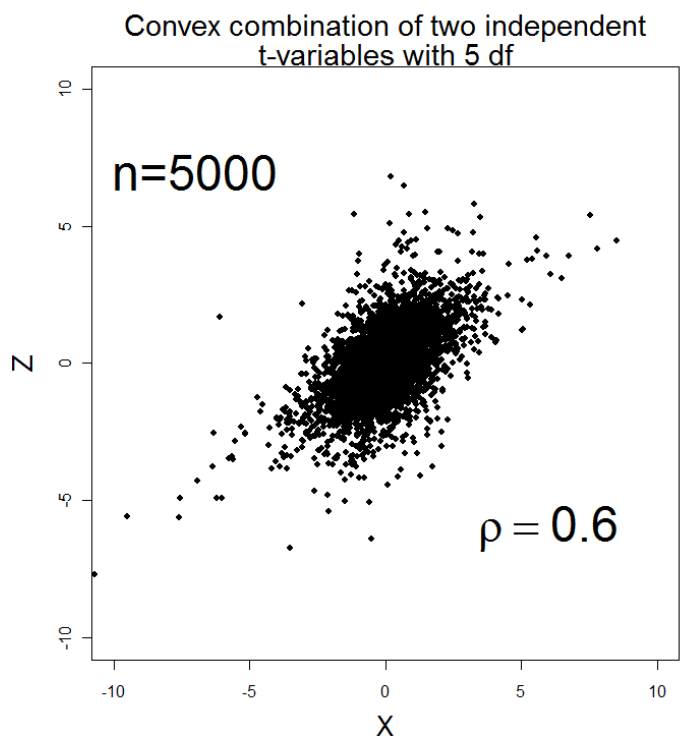

(b)

Figure 4: Joint distribution of correlated $t_{5}$-Variables 


\section{Some numerical examples}

In practice, $\rho_{X Y}$ is estimated via the empirical counterpart of $\rho_{X Y ; A}$. Solving equations (1) and (4) yields

$$
\rho_{X Y, 1}=\operatorname{sgn}\left(\rho_{X Y ; A}\right) \frac{\sigma_{X}}{\sqrt{\sigma_{X}^{2}-\sigma_{X ; A}^{2}\left(1-\frac{1}{\rho_{X Y ; A}}\right)}}
$$

and

$$
\rho_{X Y, 2}=\operatorname{sgn}\left(\rho_{X Y ; A}\right) \frac{\sigma_{Z ; A}}{\sqrt{\sigma_{Z ; A}^{2}-\sigma_{X ; A}^{2}\left(1-\frac{1}{\rho_{X Y ; A}}\right)}} .
$$

Applying (5) in the context of a bivariate $t$-distribution leads to an underestimation of the absolute values of $\rho_{X Y}$ whenever $\sigma_{Z ; A}>\sigma_{X}$. This is the case for conditions such as $A=\{X \mid X>C\}$ or $A=\{X|| X \mid>C\}$ with $C>0$.

For certain degrees of freedom, exact expressions for $\sigma_{X ; A}^{2}$ and $\sigma_{Z ; A}$ can be derived and, therefore, the difference of (6) and (5) can be calculated exactly. For a $B V T_{5}$-distribution for instance it is straightforward to show that

$$
\mathrm{E}_{X^{2} ; A}=5 / 6 \frac{\left(5+C^{2}\right)^{2} \pi+10 \sqrt{5} C-2 \sqrt{5} C^{3}-2\left(5+C^{2}\right)^{2} \arctan (C / \sqrt{5})}{P \pi\left(5+C^{2}\right)^{2}}
$$

and

$\sigma_{Z ; A}^{2}=5 / 6 \frac{5 \pi-10 \arctan (C / \sqrt{5})-2 \arctan (C / \sqrt{5}) C^{2}+\pi C^{2}-2 \sqrt{5} C}{P \pi\left(5+C^{2}\right)}$

where

$$
P=1 / 2-10 / 3 \frac{\sqrt{5} C}{\pi\left(5+C^{2}\right)^{2}}-\frac{\sqrt{5} C}{\pi\left(5+C^{2}\right)}-\frac{\arctan (C / \sqrt{5})}{\pi}
$$




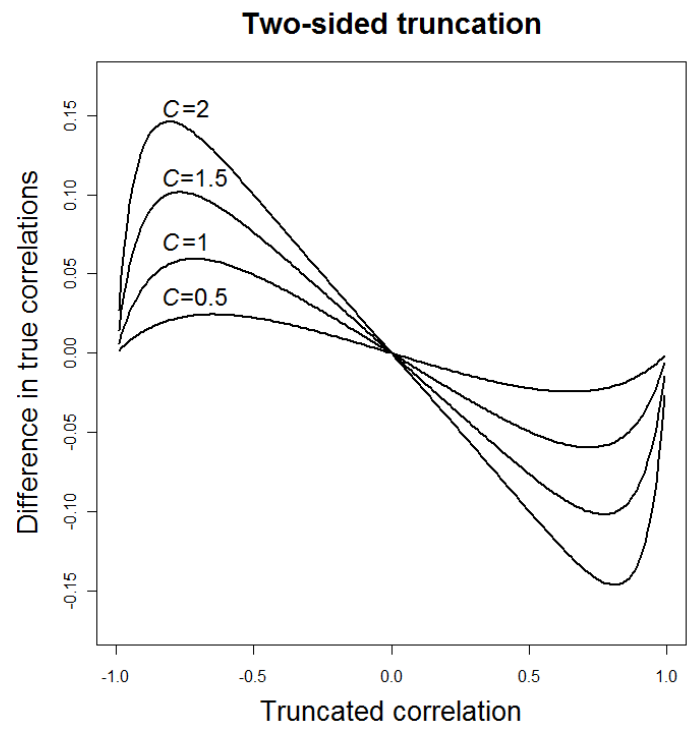

(a)

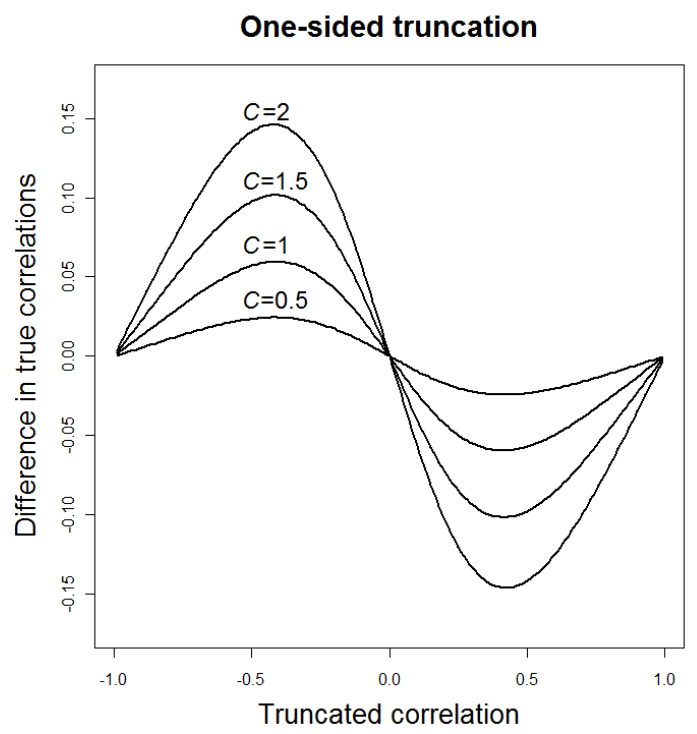

(b)

Figure 5: $\rho_{X Y, 1}-\rho_{X Y, 2}$ depending on $\rho_{X Y ; A}$

is a normalization constant. The unconditional variance is $5 / 3$. From (7) we can calculate $\sigma_{X ; A}^{2}$ for one-sided truncations via

$$
\mathrm{E}_{X ; A}=\frac{50 \sqrt{5}}{3 P \pi\left(5+C^{2}\right)^{2}}
$$

while for two-sided truncations $\mathrm{E}_{X ; A}=0$ and $\sigma_{X ; A}^{2}=\mathrm{E}_{X^{2} ; A}$.

Figure 5 plots the bias if (5) is used instead of (6) for a $B V T_{5}$-distribution as a function of $\rho_{X Y ; A}$. It shows that, for positive values of $\rho$, the application of (5) leads to an underestimation of $\rho_{X Y}$. This is especially serious for true correlations in the range of $0.4-0.7$, as shown in figure 5 , which is very common when dealing with correlations of financial returns, and for large values of the threshold $C$. The largest value $C=2$ examined here corresponds to roughly $10 \%(5 \%)$ of the observations for the two-sided (one-sided) truncation

The difference in correlations will eventually vanish for increasing degrees of freedom since the bivariate $t$-distribution then approaches the bivariate normal distribution. 


\section{References}

Bautista, C.C. (2006): "The exchange rate-interest differential relationship in six East Asian countries", Economics Letters 92, 137-142.

Bilodeau, M. and Brenner, D. (1999): Theory of Multivariate Statistics, Springer, New York.

Boyer, B.H., Gibson, M.S. and Lauretan, M. (1997): "Pitfalls in tests for changes in correlations", International Finance Discussion Paper 597, Board of the Governors of the Federal Reserve System.

Forbes, K.J. and Rigobon, R. (2002): "No Contagion, Only Interdependence: Measuring Stock Market Comovements", The Journal of Finance, 57, No. 5 , 2223-2261.

Longin, F. and Solnik, B. (1995): "Is the correlation in international equity returns constant: 1960-1990?" Journal of International Money and Finance, $14,3-26$.

Campbell, R.A., Koedijk, K.G., Kofman, P. and Forbes, C. (2008): "Increasing Correlations or Just Fat Tails?", Journal of Empirical Finance, 15, 287-309.

Johnson, N.L. and Kotz, S. (1972): Distributions in Statistics: Continuous Multivariate Distributions, Wiley, New York.

Kotz, S. and Nadarajah, S. (2004): Multivariate t Distributions and Their Applications, Cambridge University Press, Cambridge.

Solnik, B., Boucrelle, C., Fur, Y.L. (1996): "International Market Correlation and Volatility", Financial Analysts Journal, September/October, 17-34. 


